\title{
ON LENS SPACES AND THEIR SYMPLECTIC FILLINGS
}

\author{
PAOLO LisCA
}

\begin{abstract}
The standard contact structure $\xi_{0}$ on the three-sphere $S^{3}$ is invariant under the action of $\mathbb{Z} / p \mathbb{Z}$ yielding the lens space $L(p, q)$, therefore every lens space carries a natural quotient contact structure $\bar{\xi}_{0}$. A theorem of Eliashberg and McDuff classifies the symplectic fillings of $\left(L(p, 1), \bar{\xi}_{0}\right)$ up to diffeomorphism. Here we announce a generalization of that result to every lens space. In particular, we give an explicit handlebody decomposition of every symplectic filling of $\left(L(p, q), \bar{\xi}_{0}\right)$ for every $p$ and $q$. Our results imply:

(a) There exist infinitely many lens spaces $L(p, q)$ with $q \neq 1$ such that the contact 3-manifold $\left(L(p, q), \bar{\xi}_{0}\right)$ admits only one symplectic filling up to blowups and diffeomorphisms.

(b) For any natural number $N$, there exist infinitely many lens spaces $L(p, q)$ such that $\left(L(p, q), \bar{\xi}_{0}\right)$ admits more than $N$ symplectic fillings up to blowups and diffeomorphisms.
\end{abstract}

\section{Introduction}

Four-dimensional symplectic fillings are objects of central interest in symplectic and contact topology. They can be used to prove tightness of contact structures on three-dimensional manifolds [5, 10], to distinguish tight contact structures [18], and they arise in symplectic cut-and-paste constructions [23]. Symplectic fillings also have an intrinsic interest. For example, they behave like closed symplectic four-manifolds from the point of view of the Seiberg-Witten invariants and this fact has a number of consequences [14, 15, 16]. A difficult and interesting problem in this area is the diffeomorphism classification of the symplectic fillings of a given contact three-manifold. Eliashberg and McDuff solved this problem in some cases $[4,19]$. The purpose of this note is to announce a generalization of their results. A (coorientable) contact three-manifold is a pair $(Y, \xi)$, where $Y$ is a three-manifold and $\xi \subset T Y$ is a two-dimensional distribution given as the kernel of a one-form $\alpha \in \Omega^{1}(Y)$ such that $\alpha \wedge d \alpha$ is a volume form.

Received February 26, 2002.

2000 Mathematics Subject Classification. Primary 57R17; Secondary 53D35.

The author is a member of EDGE, Research Training Network HPRN-CT-2000-00101, supported by The European Human Potential Programme. The author's research was partially supported by MURST. 
A symplectic filling of a closed contact three-manifold $(Y, \xi)$ is pair $(X, \omega)$ consisting of a smooth, compact, connected four-manifold $X$ with $\partial X=Y$ and a symplectic form $\omega$ on $X$ such that $\left.\omega\right|_{\xi} \neq 0$ at every point of $\partial X$. Moreover, if $X$ is oriented by $\omega \wedge \omega$ and $\xi=\{\alpha=0\}$, the boundary orientation on $Y$ must coincide with the orientation induced by $\alpha \wedge d \alpha$ (which only depends on $\xi$ ).

A basic fact to bear in mind is that any diffeomorphism classification of symplectic fillings will always be "up to blowups". This is because a blowup, i.e. a connected sum with $\overline{\mathbb{C P}}^{2}$, is a local operation which can be performed in the symplectic category. Therefore, if $(X, \omega)$ is a symplectic filling of $(Y, \xi)$ then $\widehat{X}=X \# N \mathbb{C P}^{2}, N \geq 1$, carries a symplectic form $\widehat{\omega}$ such that $(\widehat{X}, \widehat{\omega})$ is still a symplectic filling of $(Y, \xi)$.

The standard contact structure $\xi_{0}$ on the three-sphere is the 2-dimensional distribution of complex lines tangent to $S^{3} \subset \mathbb{C}^{2}$. The unit four-ball $B^{4} \subset \mathbb{C}^{2}$ endowed with the restriction of the standard Kähler form on $\mathbb{C}^{2}$ is a symplectic filling of $\left(S^{3}, \xi_{0}\right)$. The following result, due to Eliashberg, yields the diffeomorphism classification of the symplectic fillings of $\left(S^{3}, \xi_{0}\right)$.

Theorem $1.1([4])$. Let $(X, \omega)$ be a symplectic filling of $\left(S^{3}, \xi_{0}\right)$. Then, $X$ is diffeomorphic to a blowup of $B^{4}$.

The standard contact structure $\xi_{0}$ is invariant under the natural action of $U(2)$ on $S^{3}$. Thus, $\xi_{0}$ is a fortiori invariant under the induced action of the subgroup

$$
G_{p, q}=\left\{\left(\begin{array}{cc}
\zeta & 0 \\
0 & \zeta^{q}
\end{array}\right) \mid \zeta^{p}=1\right\} \subset U(2),
$$

where $p, q \in \mathbb{Z}$. It follows that when $p>q \geq 1$ and $p, q$ are coprime, $\xi_{0}$ induces a contact structure $\bar{\xi}_{0}$ on the lens space $L(p, q)=S^{3} / G_{p, q}$.

Let $D_{p}$ denote the disk bundle over the 2 -sphere with Euler class $p$. It is not difficult to construct a symplectic form $\omega$ on $D_{-p}$ such that $\left(D_{-p}, \omega\right)$ is a symplectic filling of $\left(L(p, 1), \bar{\xi}_{0}\right)$. Another symplectic filling of $\left(L(4,1), \bar{\xi}_{0}\right)$ is given by $\mathcal{C}=\mathbb{C P}^{2} \backslash \nu(C)$, where $\nu(C)$ is a pseudo-concave neighborhood of a smooth conic $C \subset \mathbb{C P}^{2}$, endowed with the restriction of the standard Kähler form. The four-manifold $\mathcal{C}$ is easily shown to have the handlebody decomposition given by the right-hand side of Figure 2 in section 3.

The following theorem is a by-product of Dusa McDuff's classification of closed symplectic four-manifolds containing symplectic spheres of non-negative self-intersection. It yields the diffeomorphism classification of the symplectic fillings of $\left(L(p, 1), \bar{\xi}_{0}\right)$.

Theorem $1.2([19])$. Let $(X, \omega)$ be a symplectic filling of $\left(L(p, 1), \bar{\xi}_{0}\right)$. Then, $X$ is diffeomorphic to a blowup of:

(a) $D_{-p}$ if $p \neq 4$,

(b) $D_{-4}$ or $\mathcal{C}$ if $p=4$. 


\section{Symplectic fillings of $\left(L(p, q), \bar{\xi}_{0}\right)$}

From now on, we shall assume that $p$ and $q$ are two coprime integers such that

$$
p>q \geq 1, \quad \frac{p}{p-q}=b_{1}-\frac{1}{b_{2}-\frac{1}{\ddots \cdot-\frac{1}{b_{k}}}},
$$

where $b_{i} \geq 2, i=1, \ldots, k$. Note that there is a unique continued fraction expansion of $\frac{p}{p-q}$ as in $(2.1)$. We shall use the shorthand $\left[b_{1}, \ldots, b_{k}\right]$ for such continued fractions.

Let $\mathbf{n}=\left(n_{1}, \ldots, n_{k}\right)$ be a $k$-tuple of non-negative integers such that

$$
\left[n_{1}, \ldots, n_{k}\right]=0 .
$$

We can view the "thin" framed link in Figure 1 as a three-dimensional surgery presentation of a closed, oriented three-manifold $N(\mathbf{n})$. The assumption (2.2) ensures the existence of an orientation-preserving diffeomorphism

$$
\varphi: N(\mathbf{n}) \rightarrow S^{1} \times S^{2} .
$$

Definition 2.1. Let $W_{p, q}(\mathbf{n})$ be the smooth four-manifold with boundary obtained by attaching 2-handles to $S^{1} \times D^{3}$ along the framed link $\varphi(\mathbf{L}) \subset S^{1} \times S^{2}$, where $\mathbf{L} \subset N(\mathbf{n})$ is the thick framed link in Figure 1.

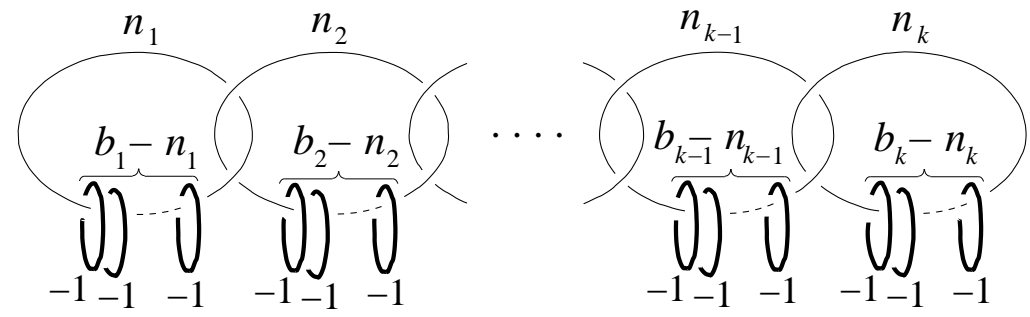

FIGURE 1. The manifold $W_{p, q}(\mathbf{n})$

Observe that the manifold $W_{p, q}(\mathbf{n})$ does not depend on the choice of the diffeomorphism $\varphi$, because every self-diffeomorphism of $S^{1} \times S^{2}$ extends to $S^{1} \times D^{3}$ [8]. Also, since $\mathbf{L}$ is canonically embedded in $S^{3}$, the framings of its components can be identified with integers.

Define $\mathbf{Z}_{p, q} \subset \mathbb{Z}^{k}$ as follows:

$$
\mathbf{Z}_{p, q}=\left\{\left(n_{1}, \ldots, n_{k}\right) \in \mathbb{Z}^{k} \mid\left[n_{1}, \ldots, n_{k}\right]=0,0 \leq n_{i} \leq b_{i}, i=1, \ldots, k\right\} .
$$

We are now ready to state our main result. 
Theorem $2.2([17])$. Let $p>q \geq 1$ be coprime integers. Then,

(a) For each $\mathbf{n} \in \mathbf{Z}_{p, q}$, the four-manifold $W_{p, q}(\mathbf{n})$ carries a symplectic form $\omega$ such that $\left(W_{p, q}(\mathbf{n}), \omega\right)$ is a symplectic filling of $\left(L(p, q), \bar{\xi}_{0}\right)$. Moreover, $W_{p, q}(\mathbf{n})$ does not contain smoothly embedded spheres with selfintersection -1 .

(b) If $(W, \omega)$ is a symplectic filling of $\left(L(p, q), \bar{\xi}_{0}\right)$, then $W$ is diffeomorphic to a blowup of $W_{p, q}(\mathbf{n})$ for some $\mathbf{n} \in \mathbf{Z}_{p, q}$.

It is interesting to compare Theorem 2.2 with properties of the versal deformations of isolated complex bidimensional singularities. The lens space $L(p, q)$, endowed with the contact structure $\bar{\xi}_{0}$, can be viewed as the link of a cyclic quotient singularity of type $(p, q)$. Stevens [22] used deep results of Kollár and Shepherd-Barron [13] to show that the irreducible components of the reduced base space $S_{\text {red }}$ of the versal deformation of such a singularity are in one-to-one correspondence with the set $\mathbf{Z}_{p, q}$ (see also [2]).

Every irreducible component of $S_{\text {red }}$ gives a smoothing of the singularity and therefore a symplectic filling of $\left(L(p, q), \bar{\xi}_{0}\right)$. It follows by Theorem 2.2 that every smoothing of a quotient $(p, q)$-singularity is diffeomorphic to a manifold of the form $W_{p, q}(\mathbf{n})$.

Indeed, it seems very likely that the smoothing coming from the irreducible component indexed by $\mathbf{n} \in \mathbf{Z}_{p, q}$ be diffeomorphic to $W_{p, q}(\mathbf{n})$. For example, it is easy to check that $\mathbf{Z}_{p, q}$ always contains the $k$-tuple $(1,2, \ldots, 2,1)$ as long as $k>1$. The corresponding irreducible component of $S_{\text {red }}$ is called the Artin component. The associated smoothing is diffeomorphic to the canonical resolution the singularity $R_{p, q}$, which is a well-known four-manifold obtained by plumbing according to a certain linear graph (see for example [1]). It is not difficult to see that $R_{p, q}$ is orientation-preserving diffeomorphic to $W_{p, q}(1,2, \ldots, 2,1)$.

If $\frac{p}{q}=\left[a_{1}, \ldots, a_{h}\right]$ with $a_{i} \geq 5, i=1, \ldots, k$, it turns out that $\mathbf{Z}_{p, q}=$ $\{(1,2, \ldots, 2,1)\}$. Thus, in this case $S_{\text {red }}$ coincides with the Artin component, a fact which had been conjectured by Kollár [12].

Under the same assumption, Theorem 2.2 implies that every symplectic filling of $\left(L(p, q), \bar{\xi}_{0}\right)$ is diffeomorphic to a blowup of $W_{p, q}(1,2, \ldots, 2,1)$. In particular, we see that there exist infinitely many lens spaces $L(p, q)$ with $q \neq 1$ such that $\left(L(p, q), \bar{\xi}_{0}\right)$ admits only one symplectic filling up to blowups and diffeomorphisms.

On the other hand, suppose that $k \geq 4, b_{2}, \ldots, b_{k-2} \geq 3$ and $b_{k} \geq k-2$. Then,

$$
\mathbf{n}(r, s)=(1, \overbrace{2, \ldots, 2}^{r}, 3, \overbrace{2, \ldots, 2}^{s}, 1, s+2) \in \mathbf{Z}_{p, q}
$$


for every $0 \leq r, s \leq k-4$ with $r+s+4=k$. Moreover, one can easily check that

$$
\operatorname{rk} H_{2}\left(W_{p, q}(\mathbf{n}(r, s)) ; \mathbb{Z}\right)=\sum_{i=1}^{k} b_{i}-2 k-s .
$$

Since the manifolds $W_{p, q}(\mathbf{n})$ do not contain exceptional spheres and therefore are not blowups of one another, Equation (2.4) implies that $\left(L(p, q), \bar{\xi}_{0}\right)$ admits at least $k-3$ symplectic fillings up to blowups and diffeomorphisms. This shows that for any natural number $N$, there exist infinitely many lens spaces $L(p, q)$ such that $\left(L(p, q), \bar{\xi}_{0}\right)$ admits more than $N$ symplectic fillings up to blowups and diffeomorphisms.

\section{Examples}

In this section we illustrate Theorem 2.2 by analyzing its implications in a few particular cases.

Given a $k$-tuple of positive integers

$$
\left(n_{1}, \ldots, n_{s-1}, n_{s}, n_{s-1}, \ldots, n_{k}\right)
$$

with $n_{s}=1$, we say that the $k-1$-tuple

$$
\left(n_{1}, \ldots, n_{s-1}-1, n_{s+1}-1, \ldots, n_{k}\right)
$$

is obtained by a blowdown at $n_{s}$ (with the obvious meaning of the notation when $s=1$ or $s=k)$. The reverse process is a blowup.

We say that a $k$-tuple of positive integers $\left(n_{1}, \ldots, n_{k}\right)$ is admissible if the continued fraction $\left[n_{1}, \ldots, n_{k}\right]$ makes sense, i.e. if none of the denominators appearing in $\left[n_{1}, \ldots, n_{k}\right]$ vanishes.

The following Lemma 3.1 can be proved by an easy induction.

Lemma 3.1. Let $\left(n_{1}, \ldots, n_{k}\right)$ be an admissible $k$-tuple of positive integers. Then, $\left[n_{1}, \ldots, n_{k}\right]=0$ if and only if $\left(n_{1}, \ldots, n_{k}\right)$ is obtained from (0) by a sequence of blowups.

First Example. Let us apply Theorem 2.2 to determine all the symplectic fillings of $\left(L(p, 1), \bar{\xi}_{0}\right)$. We have

$$
\frac{p}{p-1}=[\overbrace{2, \ldots, 2}^{p-1}] .
$$

Using Lemma 3.1 one can easily check that:

$$
\mathbf{Z}_{p, 1}=\left\{\begin{array}{l}
\{(1,2, \ldots, 2,1)\}, \text { if } p \neq 4, \\
\{(1,2,1),(2,1,2)\}, \text { if } p=4 .
\end{array}\right.
$$


Therefore, Theorem 2.2 implies that the symplectic fillings of $\left(L(p, 1), \bar{\xi}_{0}\right)$ up to blowups and diffeomorphisms are $W_{p, 1}(1,2, \ldots, 2,1)$ for $p \neq 4$ and either $W_{4,1}(1,2,1)$ or $W_{4,1}(2,1,2)$ for $p=4$.

But $W_{p, 1}(1,2, \ldots, 2,1)$ is diffeomorphic to the canonical resolution $R_{p, 1}=$ $D_{-p}$, while Figure 2 shows that $W_{4,1}(2,1,2)=\mathcal{C}$. As expected, we have just re-obtained Theorem 1.2.

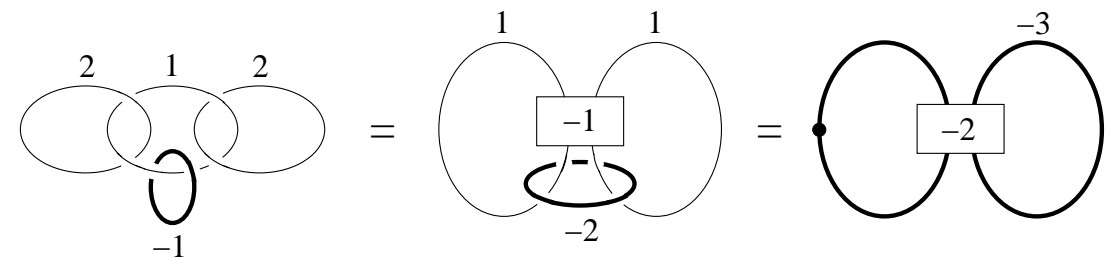

Figure 2. The manifold $W_{4,1}(2,1,2)=\mathcal{C}$

Second Example. Let us consider $L\left(p^{2}, p-1\right)$. Since

$$
\frac{p^{2}}{p^{2}-p+1}=[p, \overbrace{2, \ldots, 2}^{p}],
$$

applying Lemma 3.1 one sees that

$$
\mathbf{Z}_{p^{2}, p-1}=\{(1,2, \ldots, 2,1),(p, 1,2, \ldots, 2)\} .
$$

Thus, by Theorem 2.2 , the symplectic fillings of $\left(L\left(p^{2}, p-1\right), \bar{\xi}_{0}\right)$ are given, up to blowups and diffeomorphisms, by the canonical resolution $R_{p^{2}, p-1}$ and the manifold $W_{p^{2}, p-1}(p, 1,2, \ldots, 2)$, which is a rational homology ball as is apparent from Figure 3 . In fact, Figure 3 shows that $W_{p^{2}, p-1}(p, 1,2, \ldots, 2)$ is precisely the rational homology ball used in the symplectic rational blowdown construction [6, 23], and therefore carries a symplectic form $\omega$ such that $\left(W_{p^{2}, p-1}(p, 1,2, \ldots, 2), \omega\right)$ is a symplectic filling of $\left(L\left(p^{2}, p-1\right), \bar{\xi}_{0}\right)$.

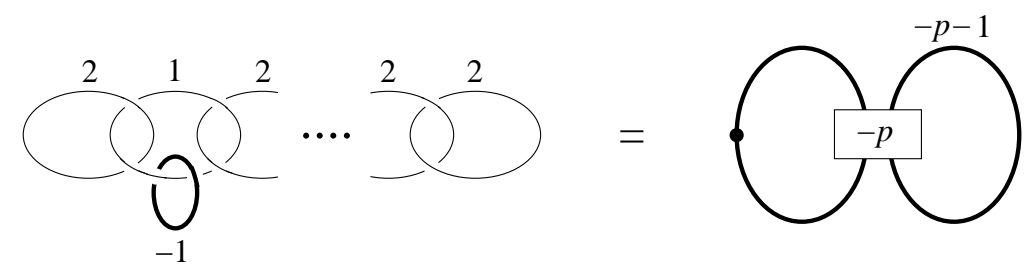

FiguRE 3. The manifold $W_{p^{2}, p-1}(p, 1,2, \ldots, 2)$

Third Example. This time we look at $L(p, p-1)$. We have $k=1$ and $\mathbf{Z}_{p, p-1}=\{(0)\}$. Hence, by Theorem 2.2(b) every filling of $L(p, p-1)$ is diffeomorphic to a blowup of $W_{p, p-1}(0)$, which is given in Figure 4. Theorem 2.2(a) 


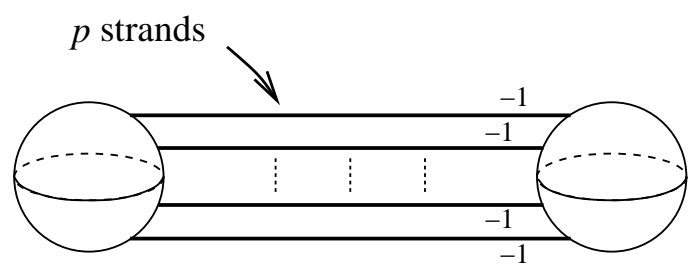

FIGURE 4. The manifold $W_{p, p-1}(0)$

says that $W_{p, p-1}(0)$ carries a symplectic structure $\omega$ such that $\left(W_{p, p-1}(0), \omega\right)$ is a symplectic filling of $\left(L(p, p-1), \bar{\xi}_{0}\right)$ and moreover $W_{p, p-1}(0)$ does not contain smoothly embedded $(-1)$-spheres. In fact, $W_{p, p-1}(0)$ is easily seen to be diffeomorphic to the canonical resolution $R_{p, p-1}$, which gives a symplectic filling of $\left(L(p, p-1), \bar{\xi}_{0}\right)$ with even intersection form.

Alternatively, using [9] one can check that $W_{p, p-1}(0)$ carries a structure of Stein surface, so in particular it carries a symplectic structure $\omega$ such that $\left(W_{p, p-1}(0), \omega\right)$ is a symplectic filling of $(L(p, p-1), \xi)$ for some contact structure $\xi$. On the other hand, on the lens space $L(p, p-1)$ there exists only one tight contact structure up to isotopy $[7,11]$. Hence, since fillable contact structures are tight we have $\xi=\bar{\xi}_{0}$ and $\left(W_{p, p-1}(0), \omega\right)$ is a symplectic filling of $\left(L(p, p-1), \bar{\xi}_{0}\right)$. As explained in the next section, the argument we have just used can be generalized to prove Theorem 2.2(a).

\section{The proof of Theorem 2.2}

In this section we outline the proof of Theorem 2.2. Complete arguments will appear elsewhere [17].

Part (a). The argument consists of two steps. First, we show that each of the manifolds $W_{p, q}(\mathbf{n})$ is a Stein surface (see e.g. [9] for the definition).

A knot $K$ in a closed, contact three-manifold $(Y, \xi)$ is called Legendrian if it is everywhere tangent to the distribution $\xi$. The contact structure induces a framing of $K$ often called the contact framing.

The standard tight contact structure $\zeta_{0}$ on $S^{1} \times S^{2}$ is the kernel of the pullback of the one-form $z d \theta+x d y-y d x$ under the inclusion $S^{1} \times S^{2} \subset S^{1} \times \mathbb{R}^{3}$, where $d \theta$ is the standard angular form on $S^{1}$ and $x, y, z$ are coordinates on $\mathbb{R}^{3}$.

The manifolds $W_{p, q}(\mathbf{n})$ are obtained by attaching 2-handles to the boundary of $S^{1} \times D^{3}$. If the 2-handles are attached along knots $K_{i}$ which are Legendrian with respect to $\zeta_{0}$ and whose framings equal their contact framings minus one, then for every choice of orientations of the $K_{i}$ 's the resulting smooth fourmanifold carries a structure of Stein surface. This construction is sometimes referred to as Legendrian surgery. It is originally due to Eliashberg [3], and 
was sistematically investigated by Gompf [9]. The first step of the proof of Theorem 2.2(a) is achieved using the following Proposition 4.1, which can be proved via an induction argument.

Proposition 4.1 ([17]). Let $\widetilde{\zeta}_{0}$ be the pull-back of $\zeta_{0}$ under the diffeomorphism (2.3) and let $\mathbf{L}$ be the "thick" framed link of Figure 1. Then, $\mathbf{L}$ is isotopic to a link $\mathcal{L}$ Legendrian with respect to $\widetilde{\zeta}_{0}$, all of whose components have contact framing equal to zero.

The existence of a Stein structure on $W_{p, q}(\mathbf{n})$ implies that $W_{p, q}(\mathbf{n})$ does not contain smoothly embedded $(-1)$-spheres. Moreover, each Stein structure induces an exact symplectic form $\omega$ on $W_{p, q}(\mathbf{n})$ and a contact structure $\xi$ on $\partial W_{p, q}(\mathbf{n})=L(p, q)$ such that $\left(W_{p, q}(\mathbf{n}), \omega\right)$ is a symplectic filling of $(L(p, q), \xi)$.

The second step of the argument consists of showing that $W_{p, q}(\mathbf{n})$ carries a Stein structure inducing a contact structure $\xi$ isomorphic to $\bar{\xi}_{0}$.

Given a 2-plane field $\xi$ on a three-manifold $M$, there is a $\operatorname{Spin}^{c}$ structure $\mathbf{s}_{\xi}$ on $M$ which only depends on $\xi$ up to homotopy. The following result is proved via an induction argument which relies on the homotopy invariant $\Gamma(\xi,-)$ introduced in $[9]$.

Proposition 4.2 ([17]). The Legendrian link $\mathcal{L}$ of Proposition 4.1 can be chosen and oriented so that the contact structure $\xi$ induced by the corresponding Stein structure satisfies $\mathbf{s}_{\xi}=\mathbf{s}_{\bar{\xi}_{0}}$.

By the classification of tight contact structures on lens spaces $[7,11], \mathbf{s}_{\xi}$ determines $\xi$ up to isotopy, hence $\xi=\bar{\xi}_{0}$. This concludes the proof of Theorem 2.2(a).

Part (b). Let $(X, \omega)$ be a symplectic four-manifold. A symplectic string in $X$ is an immersed symplectic surface

$$
\Gamma=\bigcup_{i=0}^{k} C_{i} \subset X
$$

such that:

(a) $C_{i}$ is a connected, embedded symplectic surface, $i=0, \ldots, k$;

(b) $C_{i}$ meets transversely $C_{i+1}$ at one point, $i=0, \ldots, k-1$;

(c) $C_{i} \cap C_{j}=\emptyset$ if $|i-j|>1$.

We say that $\Gamma$ as above is of type $\left(m_{0}, \ldots, m_{k}\right)$ if, furthermore,

(d) $C_{i} \cdot C_{i}=m_{i}, i=0, \ldots, k$.

Let $(W, \omega)$ be a symplectic filling of $\left(L(p, q), \bar{\xi}_{0}\right)$. Using a cut-and-paste argument which combines results from [3] and [21], it is possible to prove: 
Theorem 4.3 ([17]). There exist a symplectic four-manifold $X_{W}$ and a symplectic string

$$
\Gamma=\bigcup_{i=0}^{k} C_{i} \subset X_{W}
$$

of type $\left(1,1-b_{1},-b_{2}, \ldots,-b_{k}\right)$ such that $W$ is diffeomorphic to $X_{W} \backslash \nu(\Gamma)$, where $\nu(\Gamma) \subset X_{W}$ is a regular neighborhood of $\Gamma$.

The results of [19] imply that if $(M, \omega)$ is a closed symplectic four-manifold containing an embedded symplectic 2 -sphere $C$ of self-intersection +1 such that $M \backslash C$ is minimal (i.e. not containing embedded symplectic (-1)-spheres), then $(M, \omega)$ is symplectomorphic to $\mathbb{C P}^{2}$ with the standard Kähler structure. Morever, the symplectomorphism can be chosen so that $C$ is sent to a complex line. Thus, since non-minimal (possibly non-compact) symplectic four-manifolds can be reduced to minimal ones by blowing down exceptional symplectic spheres, applying [19] to the pair $\left(X_{W}, C_{0}\right)$ we conclude that, for some $M \geq 0$, there is a symplectomorphism

$$
\psi: X_{W} \rightarrow \mathbb{C P}^{2} \# M \overline{\mathbb{C P}}^{2}
$$

such that $\psi\left(C_{0}\right)$ is a complex line in $\mathbb{C P}^{2}$. Clearly, $\psi(\Gamma) \subset \mathbb{C P}^{2} \# M \overline{\mathbb{C P}}^{2}$ is a symplectic string of type $\left(1,1-b_{1},-b_{2}, \ldots,-b_{k}\right)$. Now we have:

Theorem $4.4([17])$. Let $\mathbb{C P}^{2} \# M \overline{\mathbb{C P}}^{2}$ be endowed with a blowup of the standard Kähler structure on $\mathbb{C P}^{2}$. Let

$$
\Delta=\bigcup_{i=0}^{k} D_{i} \subset \mathbb{C P}^{2} \# M \overline{\mathbb{C P}}^{2}
$$

be a symplectic string of type $\left(1,1-b_{1},-b_{2}, \ldots,-b_{k}\right)$ such that $D_{0} \subset \mathbb{C P}^{2}$ is a complex line. Then,

(a) $\Delta$ is the strict transform of two distinct complex linesin $\mathbb{C P}^{2}$;

(b) The complement of a regular neighborhood of $\Delta$ is diffeomorphic to $W_{p, q}(\mathbf{n})$ for some $\mathbf{n} \in \mathbf{Z}_{p, q}$.

Note that symplectic strings have well-defined stricttransforms. Part (b) of Theorem 2.2 follows immediately fromTheorem 4.4 together with the previousdiscussion. The proof of Theorem 4.4(a) relies onpositivity of intersections of $J$-holomorphic curves [20], while Theorem 4.4(b) follows directly fromTheorem 4.4(a) via a Kirby calculus argument.

\section{References}

[1] W Barth, C Peters, A Van de Ven, Compact complex surfaces, Springer-Verlag, Berlin, 1984.

[2] J A Christophersen, On the components and discriminant of the versal base space of cyclic quotient singularities, Singularity theory and its applications, Part I (Coventry, 1988/1989), 81-92, Lecture Notes in Math. 1462, Springer, Berlin, 1991. 
[3] Y Eliashberg, Topological characterization of Stein manifolds of dimension $>2$, Internat. J. of Math. 1, 1 (1990) 29-46.

[4] _ On symplectic manifolds with some contact properties, J. Differential Geom. 33 (1991) 233-238.

[5] _ Filling by holomorphic discs and its applications, London Math. Soc. Lecture Notes Series 151 (1991) 45-67.

[6] R Fintushel, R J Stern, Rational blowdowns of smooth 4-manifolds, J. Differential Geom. 46 (1997), 181-235.

[7] E Giroux, Structures de contact en dimension trois et bifurcations des feuilletages de surfaces, Invent. Math. 141 (2000) 615-689.

[8] H Gluck, The embedding of two-spheres in the four-sphere, Bull. Amer. Math. Soc. 67 (1961) 586-589.

[9] RE Gompf, Handlebody construction of Stein surfaces, Ann. of Math. (2) 148 (1998), 619-693.

[10] M Gromov,Partial differential relations, Ergebnisse der Mathematik und ihrer Grenzgebiete (3) [Results in Mathematics and Related Areas (3)], 9, Springer-Verlag, Berlin, 1986.

[11] K Honda On the classification of tight contact structures. I., Geom. Topol. 4 (2000) 309368.

[12] J Kollár, Some conjectures about the deformation space of rational surface singularities. Preprint 1988.

[13] J Kollár, N I Shepherd-Barron, Threefolds and deformations of surface singularities, Invent. Math. 91 (1988) 299-338.

[14] P B Kronheimer, TS Mrowka, Monopoles and contact structures, Invent. Math. 130 (1997), 209-256.

[15] P Lisca, Symplectic fillings and positive scalar curvature, Geom. Topol. 2 (1998) 103-116.

[16] - On symplectic fillings of 3-manifolds, in Proceedings of the $6^{\text {th }}$ Gökova Geometry-Topology Conference, Turkish J. Math. 1 (1999) 151-159.

[17] _ On symplectic fillings of lens spaces, arXiv:math.SG/0312354.

[18] P Lisca, G Matić, Tight contact structures and Seiberg-Witten invariants, Invent. Math. 129 (1997) 509-525.

[19] D McDuff, The structure of Rational and Ruled symplectic 4-manifolds, J. Amer. Math. Soc. 3 (1990) 679-712.

[20] _ Singularities and positivity of intersections of J-holomorphic curves. With an appendix by Gang Liu. Progr. Math. 117, "Holomorphic curves in symplectic geometry", 191-215, Birkhäuser, Basel, 1994.

[21] J D McCarthy, J G Wolfson, Symplectic gluing along hypersurfaces and resolution of isolated orbifold singularities, Invent. Math. 119 (1995), 129-154.

[22] J Stevens, On the versal deformation of cyclic quotient singularities, in Singularity theory and its applications, Part I (Coventry,1988/1989), 302-319, Lecture Notes in Math. 1462, Springer, Berlin, 1991.

[23] M Symington, Symplectic rational blowdowns, J. Differential Geom. 50 (1998) 505-518.

Dipartimento di Matematica, Universitì di Pisa, I-56127Pisa, itAly

E-mail address: lisca@dm.unipi.it 